\title{
Erratum: Alternative flow equation for the functional renormalization group [Phys. Rev. D 100, 101702(R) (2019)]
}

\author{
Elizabeth Alexander®, Peter Millington®, Jordan Nursey®, and Paul M. Saffin®
}

(Received 28 July 2021; published 23 September 2021)

DOI: 10.1103/PhysRevD.104.069906

We correct the functional identity reported in our paper for the inverse two-point function, as obtained from the 2PI effective action. The original conclusions are otherwise unaffected. The 2PI effective action is convex with respect to the variables $\phi_{x}^{\prime} \equiv \phi_{x}$ and $\Delta_{x y}^{\prime} \equiv \hbar \Delta_{x y}+\phi_{x} \phi_{y}$. As such, the partial functional derivatives with respect to $\phi$ that appear in Eqs. (19) and (20) of our paper are at fixed $\Delta^{\prime}$, not fixed $\Delta$. We can then show that (see Ref. [1])

$$
\begin{gathered}
\frac{\delta^{2} \Gamma^{2 \mathrm{PI}}}{\delta \phi_{x}^{\prime} \delta \phi_{z}^{\prime}} \frac{\delta^{2} W}{\delta \mathcal{J}_{z}^{\prime(k)} \delta \mathcal{J}_{y}^{(k)}}+\frac{\delta^{2} \Gamma^{2 \mathrm{PI}}}{\delta \phi_{x}^{\prime} \delta \Delta_{z w}^{\prime(k)}} \frac{\delta^{2} W}{\delta \mathcal{K}_{z w}^{\prime(k)} \delta \mathcal{J}_{y}^{\prime(k)}}=-\delta_{x y}^{(d)}, \\
\frac{\delta^{2} \Gamma^{2 \mathrm{PI}}}{\delta \phi_{x}^{\prime} \delta \phi_{u}^{\prime}} \frac{\delta^{2} W}{\delta \mathcal{J}_{u}^{\prime(k)} \delta \mathcal{K}_{y z}^{\prime(k)}}+\frac{\delta^{2} \Gamma^{2 \mathrm{PI}}}{\delta \phi_{x}^{\prime} \delta \Delta_{u v}^{\prime(k)}} \frac{\delta^{2} W}{\delta \mathcal{K}_{u v}^{\prime(k)} \delta \mathcal{K}_{y z}^{(k)}}=0, \\
\frac{\delta^{2} \Gamma^{2 \mathrm{PI}}}{\delta \Delta_{x y}^{\prime(k)} \delta \phi_{u}^{\prime}} \frac{\delta^{2} W}{\delta \mathcal{J}_{u}^{\prime(k)} \delta \mathcal{J}_{z}^{\prime(k)}}+\frac{\delta^{2} \Gamma^{2 \mathrm{PI}}}{\delta \Delta_{x y}^{\prime(k)} \delta \Delta_{u v}^{\prime(k)}} \frac{\delta^{2} W}{\delta \mathcal{K}_{u v}^{\prime(k)} \delta \mathcal{J}_{z}^{\prime(k)}}=0, \\
\frac{\delta^{2} \Gamma^{2 \mathrm{PI}}}{\delta \Delta_{x y}^{\prime(k)} \delta \Delta_{u v}^{\prime(k)}} \frac{\delta^{2} W}{\delta \mathcal{K}_{u v}^{\prime(k)} \delta \mathcal{K}_{z w}^{\prime(k)}}+\frac{\delta^{2} \Gamma^{2 \mathrm{PI}}}{\delta \Delta_{x y}^{\prime(k)} \delta \phi_{u}^{\prime}} \frac{\delta^{2} W}{\delta \mathcal{J}_{u}^{\prime(k)} \delta \mathcal{K}_{z w}^{\prime(k)}}=-\frac{1}{2}\left(\delta_{x z}^{(d)} \delta_{y w}^{(d)}+\delta_{x w}^{(d)} \delta_{y z}^{(d)}\right),
\end{gathered}
$$

in $d$ spacetime dimensions, where $\mathcal{J}^{\prime} \equiv \mathcal{J}, \mathcal{K}^{\prime} \equiv \mathcal{K} / 2, \Gamma^{2 \mathrm{PI}} \equiv \Gamma^{2 \mathrm{PI}}\left[\phi, \Delta^{(k)}\right]$ and $W \equiv W\left[\mathcal{J}^{(k)}, \mathcal{K}^{(k)}\right]$. Using

$$
\begin{gathered}
\frac{\delta}{\delta \phi_{x}^{\prime}}=\frac{\delta}{\delta \phi_{x}}-\frac{2}{\hbar} \phi_{y} \frac{\delta}{\delta \Delta_{y x}^{(k)}}, \\
\frac{\delta}{\delta \Delta_{x y}^{\prime(k)}}=\frac{1}{\hbar} \frac{\delta}{\delta \Delta_{x y}^{(k)}},
\end{gathered}
$$

as well as the other identities listed in the third to sixth rows of Table I, Eq. (1) can be written (see Ref. [1])

$$
\begin{gathered}
\left\{\frac{\delta^{2} \Gamma^{2 \mathrm{PI}}}{\delta \phi_{x} \delta \phi_{z}}-\mathcal{K}_{x z}^{(k)}-\frac{4 \phi_{w}}{\hbar}\left[\frac{\delta^{2} \Gamma^{2 \mathrm{PI}}}{\delta \phi_{(x} \delta \Delta_{z) w}}-\frac{\phi_{u}}{\hbar} \frac{\delta^{2} \Gamma^{2 \mathrm{PI}}}{\delta \Delta_{w x} \delta \Delta_{u z}}\right]\right\} \Delta_{z y}-\frac{2}{\hbar}\left[\frac{\delta^{2} \Gamma^{2 \mathrm{PI}}}{\delta \phi_{x} \delta \Delta_{z w}}-\frac{2 \phi_{u}}{\hbar} \frac{\delta^{2} \Gamma^{2 \mathrm{PI}}}{\delta \Delta_{u x} \delta \Delta_{z w}}\right] \frac{\delta^{2} W}{\delta \mathcal{J}_{y} \delta \mathcal{K}_{z w}}=\delta_{x y}^{(d)}, \\
\left\{\frac{\delta^{2} \Gamma^{2 \mathrm{PI}}}{\delta \phi_{x} \delta \phi_{z}}-\mathcal{K}_{x z}^{(k)}-\frac{4 \phi_{w}}{\hbar}\left[\frac{\delta^{2} \Gamma^{2 \mathrm{PI}}}{\delta \phi_{(x} \delta \Delta_{z) w}}-\frac{\phi_{u}}{\hbar} \frac{\delta^{2} \Gamma^{2 \mathrm{PI}}}{\delta \Delta_{w x} \delta \Delta_{u z}}\right]\right\} \frac{\delta^{2} W}{\delta \mathcal{J}_{z} \delta \mathcal{K}_{v y}}+\frac{2}{\hbar}\left[\frac{\delta^{2} \Gamma^{2 \mathrm{PI}}}{\delta \phi_{x} \delta \Delta_{z w}}-\frac{2 \phi_{u}}{\hbar} \frac{\delta^{2} \Gamma^{2 \mathrm{PI}}}{\delta \Delta_{u x} \delta \Delta_{z w}}\right] \frac{\delta^{2} W}{\delta \mathcal{K}_{v y} \delta \mathcal{K}_{z w}}=0, \\
{\left[\frac{\delta^{2} \Gamma^{2 \mathrm{PI}}}{\delta \phi_{w} \delta \Delta_{x y}}-\frac{2 \phi_{u}}{\hbar} \frac{\delta^{2} \Gamma^{2 \mathrm{PI}}}{\delta \Delta_{u w} \delta \Delta_{x y}}\right] \Delta_{w z}-\frac{2}{\hbar} \frac{\delta^{2} \Gamma^{2 \mathrm{PI}}}{\delta \Delta_{w u} \delta \Delta_{x y}} \frac{\delta^{2} W}{\delta \mathcal{J}_{z} \delta \mathcal{K}_{w u}}=0,}
\end{gathered}
$$

Published by the American Physical Society under the terms of the Creative Commons Attribution 4.0 International license. Further distribution of this work must maintain attribution to the author(s) and the published articles title, journal citation, and DOI. 
TABLE I. Reproduction of Table I of our paper wherein the expression for the inverse two-point function $\Delta_{x y}^{(k),-1}$ in the 2 PI case has been corrected. Note that, as in our paper, we use $\mathcal{K}^{(k)}$ and $\mathcal{R}^{(k)}$ interchangeably in the 2PI case.

\begin{tabular}{|c|c|}
\hline Average 1PI & $2 \mathrm{PI}$ \\
\hline$\Gamma_{\mathrm{av}}^{1 \mathrm{PI}}\left[\phi, \mathcal{R}^{(k)}\right]=W\left[\mathcal{J}^{(k)}[\phi], \mathcal{R}^{(k)}\right]+\mathcal{J}_{x}^{(k)}[\phi] \phi_{x}+\frac{1}{2} \mathcal{R}_{x y}^{(k)} \phi_{x} \phi_{y}$ & $\begin{array}{l}\Gamma^{2 \mathrm{PI}}\left[\phi, \Delta^{(k)}\right]=W\left[\mathcal{J}^{(k)}\left[\phi, \Delta^{(k)}\right], \mathcal{K}^{(k)}\left[\phi, \Delta^{(k)}\right]\right] \\
+\mathcal{J}_{x}\left[\phi, \Delta^{(k)}\right] \phi_{x}+\frac{1}{2} \mathcal{K}_{x y}^{(k)}\left[\phi, \Delta^{(k)}\right]\left(\phi_{x} \phi_{y}+\hbar \Delta_{x y}^{(k)}\right)\end{array}$ \\
\hline$\phi_{x}=-\frac{\delta W\left[\mathcal{J}^{(k)}[\phi], \mathcal{R}^{(k)}\right]}{\delta \mathcal{J}_{x}^{(k)}[\phi]}$ & $\phi_{x}=-\frac{\delta W\left[\mathcal{J}^{(k)}\left[\phi, \Delta^{(k)}\right], \mathcal{K}^{(k)}\left[\phi, \Delta^{(k)}\right]\right]}{\delta \mathcal{J}_{x}^{(k)}\left[\phi, \Delta^{(k)}\right]}$ \\
\hline $\begin{array}{l}\hbar \Delta_{x y}^{(k)}=-2 \frac{\delta W\left[\mathcal{J}^{(k)}[\phi], \mathcal{R}^{(k)}\right]}{\delta \mathcal{R}_{x y}^{(k)}}-\phi_{x} \phi_{y} \\
\quad=-\hbar \frac{\delta^{2} W\left[\mathcal{J}^{(k)}[\phi], \mathcal{R}^{(k)}\right]}{\delta \mathcal{J}_{x}^{(k)}[\phi] \delta \mathcal{J}_{y}^{(k)}[\phi]}\end{array}$ & $\begin{aligned} \hbar \Delta_{x y}^{(k)}= & -2 \frac{\delta W\left[\mathcal{J}^{(k)}\left[\phi, \Delta^{(k)}\right], \mathcal{K}^{(k)}\left[\phi, \Delta^{(k)}\right]\right]}{\delta \mathcal{K}_{x y}^{(k)}\left[\phi, \Delta^{(k)}\right]}-\phi_{x} \phi_{y} \\
& =-\hbar \frac{\delta^{2} W\left[\mathcal{J}^{(k)}\left[\phi, \Delta^{(k)}\right], \mathcal{K}^{(k)}\left[\phi, \Delta^{(k)}\right]\right]}{\delta \mathcal{J}_{x}^{(k)}\left[\phi, \Delta^{(k)}\right] \delta \mathcal{J}_{y}^{(k)}\left[\phi, \Delta^{(k)}\right]}\end{aligned}$ \\
\hline$\frac{\delta \Gamma_{\mathrm{av}}^{\mathrm{PP}}\left[\phi, \mathcal{R}^{(k)}\right]}{\delta \phi_{x}}=\mathcal{J}_{x}^{(k)}[\phi]+\mathcal{R}_{x y}^{(k)} \phi_{y}$ & $\frac{\delta \Gamma^{2 \mathrm{PI}}\left[\phi, \Delta^{(k)}\right]}{\delta \phi_{x}}=\mathcal{J}_{x}^{(k)}\left[\phi, \Delta^{(k)}\right]+\mathcal{K}_{x y}^{(k)}\left[\phi, \Delta^{(k)}\right] \phi_{y}$ \\
\hline$\frac{\delta \Gamma_{a \mathrm{v}}^{1 \mathrm{PI}}\left[\phi, \mathcal{R}^{(k)}\right]}{\delta \mathcal{R}_{x y}^{(k)}}=-\frac{\hbar}{2} \Delta_{x y}^{(k)}$ & $\frac{\delta \Gamma^{2 \mathrm{PP}[}\left[\phi, \Delta^{(k)}\right]}{\delta \Delta_{x y}^{(k)}}=+\frac{\hbar}{2} \mathcal{K}_{x y}^{(k)}\left[\phi, \Delta^{(k)}\right]$ \\
\hline $\begin{array}{l}\Delta_{x y}^{(k),-1}=\frac{\delta^{2} \Gamma^{\mathrm{IP}}\left[\phi, \mathcal{R}^{(k)}\right]}{\delta \phi_{x} \delta \phi_{y}} \\
\quad=\frac{\delta^{2} \Gamma_{a \gamma}^{\mathrm{PP}}\left[\phi, \mathcal{R}^{(k)}\right]}{\delta \phi_{x} \delta \phi_{y}}-\mathcal{R}_{x y}^{(k)} \\
\quad=S_{x y}^{(2)}[\phi]-\mathcal{R}_{x y}^{(k)}+\mathcal{O}(\hbar)\end{array}$ & $\begin{array}{l}\Delta_{x y}^{(k),-1}=\frac{\delta^{2} \Gamma^{2 \mathrm{PP}}\left[\phi, \Delta^{(k)}\right]}{\delta \phi_{x} \delta \delta y}-\mathcal{K}_{x y}^{(k)}\left[\phi, \Delta^{(k)}\right] \\
-\frac{\delta^{2} \Gamma^{2 \mathrm{PI}}\left[\phi, \Delta^{(k)}\right]}{\delta \phi_{x} \delta \Delta_{z w}^{(k)}}\left(\frac{\delta^{2} \Gamma^{2 \mathrm{PI}}\left[\phi, \Delta^{(k)}\right]}{\partial \Delta_{z w}^{(k)} \delta \Delta_{u v}^{(k)}}\right)^{-1} \frac{\delta^{2} \Gamma^{2 \mathrm{PI}}\left[\phi, \Delta^{(k)}\right]}{\delta \Delta_{u v}^{(k)} \delta \phi_{y}} \\
\quad=S_{x y}^{(2)}[\phi]-\mathcal{K}_{x y}^{(k)}\left[\phi, \Delta^{(k)}\right]+\mathcal{O}(\hbar)\end{array}$ \\
\hline$\partial_{k} \Gamma_{\mathrm{av}}^{1 \mathrm{PI}}\left[\phi, \mathcal{R}^{(k)}\right]=\frac{\delta \Gamma_{\mathrm{av}}^{[\mathrm{P}[}\left[\phi, \mathcal{R}^{(k)}\right]}{\delta \phi_{x}} \partial_{k} \phi_{x}+\frac{\delta \Gamma_{\mathrm{av}}^{\mid \mathrm{P} \mathrm{P}}\left[\phi, \mathcal{R}^{(k)}\right]}{\delta \mathcal{R}_{x y}^{(k)}} \partial_{k} \mathcal{R}_{x y}^{(k)}$ & $\partial_{k} \Gamma^{2 \mathrm{PI}}\left[\phi, \Delta^{(k)}\right]=\frac{\delta \Gamma^{2 \mathrm{PI}[}\left[\phi, \Delta^{(k)}\right]}{\delta \phi_{x}} \partial_{k} \phi_{x}+\frac{\delta \Gamma^{2 \mathrm{PP}[}\left[\phi, \Delta^{(k)}\right]}{\delta \Delta_{x y}^{(k)}} \partial_{k} \Delta_{x y}^{(k)}$ \\
\hline
\end{tabular}

$$
\frac{2}{\hbar}\left\{\left[\frac{\delta^{2} \Gamma^{2 \mathrm{PI}}}{\delta \phi_{u} \delta \Delta_{x y}}-\frac{2 \phi_{v}}{\hbar} \frac{\delta^{2} \Gamma^{2 \mathrm{PI}}}{\delta \Delta_{v u} \delta \Delta_{x y}}\right] \frac{\delta^{2} W}{\delta \mathcal{J}_{u} \delta \mathcal{K}_{z w}}+\frac{2}{\hbar} \frac{\delta^{2} \Gamma^{2 \mathrm{PI}}}{\delta \Delta_{x y} \delta \Delta_{u v}} \frac{\delta^{2} W}{\delta \mathcal{K}_{u v} \delta \mathcal{K}_{z w}}\right\}=-\frac{1}{2}\left(\delta_{x z}^{(d)} \delta_{y w}^{(d)}+\delta_{x w}^{(d)} \delta_{y z}^{(d)}\right),
$$

as appeared (in condensed notation) in footnote 11 of Ref. [2]. Herein, we have omitted the superscript " $(k)$ " on $\Delta, \mathcal{J}$, and $\mathcal{K}$, and used the shorthand notation $A_{(x} B_{z) w} \equiv \frac{1}{2}\left(A_{x} B_{z w}+A_{z} B_{x w}\right)$ for symmetrization in coordinates. Derivatives with respect to $\phi$ are at fixed $\Delta$ and vice versa; derivatives with respect to $\mathcal{J}$ are at fixed $\mathcal{K}$ and vice versa. Note that $\delta^{2} W / \delta \mathcal{J}_{x}^{(k)} / \delta \mathcal{K}_{y z}^{(k)}=-\delta \phi_{x} / \delta \mathcal{K}_{y z}^{(k)} \neq 0$ (even though $\partial_{k} \phi=0$ ), as was erroneously assumed in our paper, leading to the incorrect identity in Eq. (21). Additionally, while the restriction on $\mathcal{K}_{x y} \equiv \mathcal{K}_{x y}^{(k)}=\mathcal{R}_{x y}^{(k)}$ fixes $\Delta_{x y} \equiv \Delta_{x y}^{(k)}$ to be a functional of $\phi$ (for clarification, see Ref. [1]), the partial functional derivative of $\Delta$ with respect to $\phi$ at fixed $\Delta$ remains zero by definition, and we retract the potentially misleading notation $\Delta_{x y}^{(k)}[\phi]$ used in our paper.

By solving Eq. (3), we can show (see Ref. [1]) that the inverse two-point function is given by

$$
\Delta_{x y}^{(k),-1}=\frac{\delta^{2} \Gamma^{2 \mathrm{PI}}}{\delta \phi_{x} \delta \phi_{y}}-\mathcal{K}_{x y}^{(k)}-\frac{\delta^{2} \Gamma^{2 \mathrm{PI}}}{\delta \phi_{x} \delta \Delta_{z w}^{(k)}}\left(\frac{\delta^{2} \Gamma^{2 \mathrm{PI}}}{\partial \Delta_{z w}^{(k)} \delta \Delta_{u v}^{(k)}}\right)^{-1} \frac{\delta^{2} \Gamma^{2 \mathrm{PI}}}{\delta \Delta_{u v}^{(k)} \delta \phi_{y}} .
$$

Equation (4) replaces Eq. (21) of our paper, and the seventh row of Table I of our paper should be corrected as shown in Table I of this erratum, where we have reproduced the complete table from our paper for clarity.

This work was supported by a Nottingham Research Fellowship from the University of Nottingham; the Leverhulme Trust [Grant No. RL-2016-028]; and the Science and Technology Facilities Council [Grant No. ST/P000703/1]. The Authors would like to thank Dario Benedetti, Kevin Falls, Jan Pawlowski and Adam Rancon for discussions at the 10th International Conference on Exact Renormalization Group 2020 (ERG2020), hosted by the Yukawa Institute for Theoretical Physics, Japan, which led to the corrections and clarifications herein.

[1] P. Millington and P. M. Saffin, Benchmarking regulator-sourced 2PI and average 1PI flow equations in zero dimensions, arXiv:2107.12914.

[2] J. M. Cornwall, R. Jackiw, and E. Tomboulis, Effective action for composite operators, Phys. Rev. D 10, 2428 (1974). 